\title{
Investigation of plant flavonoids as potential dengue protease inhibitors
}

\author{
Mangala K Jayadevappa ${ }^{1,2}{ }^{\circledR}$, Pooja R Karkera ${ }^{2,3}{ }^{\circledR}$, Ravikumar Y Siddappa $^{2^{*} \mathbb{D}}$, Sandeep Telkar ${ }^{\circledR}$, Prashantha \\ Karunakara $^{5}$ \\ ${ }^{1}$ Department of Biotechnology, Bharathiar University, Coimbatore, Tamil Nadu-641046, India \\ ${ }^{2}$ Department of Biotechnology, M. S. Ramaiah Institute of Technology, Bengaluru-560 054, Karnataka, India \\ ${ }^{3}$ Department of Biochemistry, REVA University, Bengaluru 560064, Karnataka, India \\ ${ }^{4}$ Department of Biotechnology, Jnanasayadri, Kuvempu University, Shankaraghatta-577129, Shivamogga Dst., Karnataka, India \\ ${ }^{5}$ Department of Biotechnology, PES University, Bengaluru 560085, Karnataka, India
}

\section{A R T I C L E I N F O}

\section{Article Type:}

Original Article

\section{Article History:}

Received: 12 November 2019

Accepted: 10 March 2020

\section{Keywords:}

Flavonoids

NS2/NS3 protease

Dengue virus

Protease inhibitors

Molecular docking

\begin{abstract}
A B S T R A C T
Introduction: Dengue infection is one of the worldwide problems with an increasing rate. Dengue virus (DENV) belonging to Flaviviridae family carries a positive single strand RNA, consists of four serotypes, gives rise to dengue shock syndrome, undifferentiated fever (DF), and dengue hemorrhagic fever. Unfortunately, there is no licensed drug available for various serotypes and the vaccine candidates are still under investigation. Flavonoids are a group of important phytochemicals of many plants used against various viruses. Therefore, the present study was designed to screen the subset of plant flavonoids against the DENV NS2/ NS3 protease of 3L6P and 2VBC of serotype 1 and serotype 4 respectively by in silico docking. Methods: Flavonoids were subjected to docking study using AutoDock Vina. The lead molecule was further investigated for its dengue viral protease inhibition. Further the cytotoxicity of lead molecule was tested by 3-(4,5-dimethylthiazol-2-yl)-2,5-diphenyltetrazolium bromide (MTT) assay. Absorption, distribution, metabolism, excretion and toxicity (ADMET) study was performed using OSIRIS software.

Results: The flavonoids amentoflavone, quercetin-3-O- $\beta$-D-glucopyranoside, avicularin, reynoutrin, silymarin and scutallarein showed potential interactions in both serotype 1 and serotype 4 and hence these flavonoids can be served as potential lead candidates to stop viral replication. Amentoflavone, the lead molecule, showed potential in-vitro protease inhibition activity and also the concentration was below the minimal effective level for the in-vitro cytotoxicity test conducted.

Conclusion: This finding has laid the groundwork for eventual identification of these flavonoids in developing potential inhibitor candidates for further development by in-vitro extraction and chemical modification methods.
\end{abstract}

Implication for health policy/practice/research/medical education:

Amentoflavone, the lead molecule, could be a potential inhibitor of dengue viral NS2B/NS3 protease enzyme. Further investigation may lead to develop amentoflavone as drug candidate to treat dengue infection.

Please cite this paper as: Jayadevappa MK, Karkera PR, Siddappa RY, Telkar S, Karunakara P. Investigation of plant flavonoids as potential dengue protease inhibitors. J Herbmed Pharmacol. 2020;9(4):366-373. doi: 10.34172/jhp.2020.46.

\section{Introduction}

Dengue is one of the most prevalent mosquito borne viral infection in the world (1-3). It has become a global health problem affecting almost 2.5 billion people (4) with more than 25000 deaths per year (5). It is transmitted by Aedes aegypti mosquitoes and affects tropical and subtropical countries. Dengue virus (DENV) is a member of Flaviviridae family and contains four serotypes including DENV-1, DENV-2, DENV-3 and DENV-4 (6). In spite of high incidence severity and economic burden, there is no available licensed drug for various serotypes and the vaccine candidates are still under investigation. 
Development of an efficient vaccine faces the challenge to provide protection against all four serotypes at once, otherwise it might render the immunized individuals more susceptible to undifferentiated fever (7).

The DENV genome encodes a polyprotein, which is cleaved into three structural and seven non-structural proteins (8). NS3 protease is an important target to design new antiviral drugs against DENV. Hence, designing potent inhibitors against DENV NS3 seems to be a good idea (9). Despite approaches such as RNAi technology and monoclonal antibodies for this purpose, there is still no licensed drug or vaccine available for dengue (10). At present, only supportive therapies usually used $(11,12)$. Plant remedies, due to low side effects and high accessibility are considered as an important source for development of new drugs (13-21). They contain various phytochemicals such as flavonoids, alkaloids, polyenes, polyphenolics, peptides, limonoids, and saponins revealing therapeutic viral entry and DNA/RNA replication against diverse range of viruses (10).

Flavonoids are secondary metabolites of plants with low molecular-weight existing ubiquitously in various plants in forms of aglycones, glycosides, and methylated derivatives (22). Flavonoids are used in traditional Eastern medicine and have anti-oxidant, anti-tumor, anti-proliferative, antiinflammatory, and pro-apoptotic activities (23-26).

Recent computational drug-design approaches have opened new doors to help obtain new leads in drug development studies. Molecular docking technique allows screening thousands of compounds and selecting few compounds which could be later subjected to further study by extraction methods. Therefore, the present study was designed to screen 107 flavonoids from about 47 plants listed against DENV NS2B/NS3 protease by in silico techniques.

Amentoflavone is a common biflavonoid which naturally occurs in many plants. Bioactivities of Amentoflavone including anti-oxidant, anti-inflammatory, anti-tumor, anti-virus, anti-fungal effects have been carried out but no study has been conducted on its anti-Dengue property. Therefore, the present study aims also to find its potential dengue protease inhibition property.

\section{Materials and Methods}

In the present study 107 flavonoids were docked against DENV NS2/NS3 protease 3L6P and 2VBC of serotypes 1 and 4 , respectively.

\section{Criteria for the selection of plants}

Forty-seven plants were selected for the study by using the following criteria: Plant extracts that reported antiviral effect, plants used by traditional medical practitioners like Ayurveda and Chinese medicine and plants reported to inhibit viral protease enzyme (eg. Dengue, hepatitis C virus protease).
Selection of compounds

From the list of selected plants using the criteria mentioned above, the flavonoids that were reported in these plants were searched in online tools using search engines such as NCBI, BMC, and Science Direct.

\section{Ligand preparation}

A structure-based approach was followed to design the inhibitors. From the list of selected plants using the criteria mentioned above, the flavonoids reported in these plants were searched in online tools using search engines such as NCBI, BMC and Science Direct. Chemical structures of the flavonoids were downloaded from PubChem and the $2 \mathrm{D}$ structure of each of them was drawn using ChemDraw Ultra version 6.0.1 software and saved in .cdx format. These $2 \mathrm{D}$ structures were converted into $3 \mathrm{D}$ structures and optimized by minimizing their energy using Chem 3D Ultra version 6.0 software and saved in .pdb format and used as input file for opening in AutoDock. The molecules were opened in PyRx software, converted and saved in .pdbqt format which was further used for docking.

\section{Refinement of receptor protein}

The 3D structure of DENV NS2B/NS3 protease of serotypes 1 and 4 were retrieved from Protein Data Bank(PDB) using PDB ID: 3L6P and 2VBC, respectively (http:/www.rcsb.org/pdb) in .pdb format. This was opened in AutoDock and optimized by deleting the water molecule, removing the heteroatoms etc. and was saved in .pdbqt format. This minimized structure was used as receptor for docking studies.

The grid box (having conformation: centre $\mathrm{x}=-23.5270$, center $y=-19.2167$, center $z=27.1656$ ) was chosen for the protein 3L6P on their active sites (Ile-75, Arg-104, Gly-105, Pro-117, Trp-119, Asp-125, Tyr-129, Gly-130, Gly-131, Trp-133, Phe-135, Trp-139, Pro-152, Gln-160, Pro-163, Gly-174, Asp-179, Gly-183, Ser-185, Tyr-200, Gly-201, Asn-202, Gly-203, Val-212, Ser-213, Ile-215, Gln-217)

The grid box (having conformation: centre $x=-9.8210$, center $y=3.5911$, center $z=-0.7172$ ) was chosen for the protein 2VBC on their active sites (Asp-75, Lys-104, Asn105, Lys-117, Leu-119, Ala-125, Asp-129, Phe-130, Lys131, Gly-133, Ser-135, Ile-139, Asn-152, Asp-160, Ser-163, Pro-174, Asp-179, Phe-183, Lys-185, Thr-200, Lys-201, Arg-202, Ile-203, Leu-212, Lys-213, Arg-215, Arg-217).

Molecular docking

Molecular docking of the flavonoids with NS2B/NS3 protease was carried out by using AutoDock Tools version 1.5.6. The best ranked models with the lowest binding energy were analyzed further and visualized using LIGPLOT software.

ADMET study

Absorption, distribution, metabolism, excretion, 
toxicology (ADMET) study was performed in silico for all the 107 flavonoids by using the OSIRIS software. The drug-likeliness and toxicity of the compounds irrespective of the protein was determined.

\section{Protease assay}

The lead molecule, amentoflavone was investigated for dengue viral protein inhibition using high-throughput protease assay (28). The reaction mixture containing 40 $\mu \mathrm{L}$ protease buffer of $\mathrm{pH}-9,1 \mu \mathrm{L}$ of enzyme and varying concentration $(5-25 \mu \mathrm{M})$ of amentoflavone (working standard-5 $\mu \mathrm{g} / \mu \mathrm{L}$ ) was pre-incubated for 15 minutes at $37^{\circ} \mathrm{C}$. Subsequently $1 \mu \mathrm{L}$ of substrate was added to each reaction mixture and incubated at same temperature for 1 hour. After incubation, fluorescence was measured using excitation filter $485 \mathrm{~nm}$ and emission filter $538 \mathrm{~nm}$ in a fluorometer (Modulus ${ }^{\mathrm{TM}}$ Microplate Multimode reader). The difference between the fluorescence obtained in the presence and absence of the compound was taken as the measure of the inhibitory activity. Quercetin was used as positive control.

\section{Cytotoxicity assay}

HepG2 cells ( $10^{5}$ cells/well in 96 well plate) were cultured in Dulbecco's Modified Eagle Medium (DMEM) containing with glutamine $(0.6 \mathrm{~g} / \mathrm{L})$, gentamicin $(25 \mathrm{mg} /$ $\mathrm{ml}$ ) and $10 \%$ fetal calf serum at $37^{\circ} \mathrm{C}$ and humidified with $5 \% \mathrm{CO}_{2}$. After 24 hours, the medium was replaced with fresh media containing compound $(5,10,15,20$ and $25 \mu \mathrm{M})$ dissolved in dimethyl sulfoxide (DMSO) (1\%) was added to each well and incubated for 72 hours. At the end of 72 hours incubation, the medium in each well was again replaced with fresh medium containing $0.5 \mathrm{mg} / \mathrm{mL}$ of 3-[4,5-dimethylthiazole-2-yl]-2,5-diphenyltetrazolium bromide (MTT) and further incubated for 4 hours. MTT containing media was replaced with $100 \mu \mathrm{L}$ of DMSO and optical density (OD) was recorded at $570 \mathrm{~nm}$ (29). The cells without compound treatment were taken as blank and the cell viability was calculated using the following formula:

$\%$ Viable cell $=(\mathrm{OD}$ of Test/OD of Control $) \times 100$

\section{Results}

Selection of plants

Based on the biological significance, 47 plants were selected which have shown anti-viral effect and viral protease inhibitory activities (remaining compounds, their plants source and docking score against both the protease are reported in Table S1) (Table 1).

Results of molecular docking study

The $3 \mathrm{D}$ structure of the DENV proteases was retrieved from PDB. The PDB ID of 3D structure were 3L6P and $2 \mathrm{VBC}$ respectively for serotype 1 and serotype 4 . Molecular docking was performed for 107 flavonoids listed from the selected plants with both serotypes. Flavonoids, which showed least binding energy with highest stability were selected and subjected for Ligplot analysis (Table 2).

Ligplot analysis of lead molecules with protease enzyme of serotype 1 (3L6P)

In this study, in silico investigation results revealed that among all the docked flavonoids against 3L6P, amentoflavone (hydrophobic interactions- Gly-20, Asp125, Lys-124, Ala-214, Ala-216, Val-175 hydrophilic interactions- Asn-202, Lys-123, Ile-215, Gln-217) was ranked first followed by quercetin-3-O- $\beta-\mathrm{D}$ glucopyranoside (hydrophobic interactions- Tyr-211, Leu178, Thr-184, Ser-185, Pro-182 hydrophilic interactionsGly-183, Lys-181, Phe-180, Asn-202, Tyr-200, Asp-179); liquiritinapioside (hydrophobic interactions- Ser-185, Pro-182, Asn-202, Gly-203, Val-212, Tyr-211, Ser-213 hydrophilic interactions- Lys-181, Gly-183, Thr-184, Leu-178, Tyr-250); Silybin (hydrophobic interactions -Tyr-211, Tyr-200, Gly-183, Asn-202, Phe-180, Pro-182, Lys-181, Thr-184, Ser-185 hydrophilic interactions Leu-178, Asp-179, Gly-203, Asp-125, His-101, Val-86 ); Avicularin (hydrophobic interactions- Ser-185, Pro-182, Tyr-200, Gly-203, Ser-213, Tyr-211, Leu-178, Gly-201 hydrophilic interactions- Gly-183, Lys-181, Phe-180, Asn202, Asp-179); reynoutrin (hydrophobic interactionsTyr-200, Ser-185, Asn-202, Gly-203, Ser-213,Val-212, Leu-178, Tyr-211, Pro-182 hydrophilic interactions- Gly-

Table 1. Lead molecule and its plant source

\begin{tabular}{|c|c|c|}
\hline Flavonoids & Plant source & Ref. \\
\hline Amentoflavone & Selaginella tamariscina & $(30)$ \\
\hline Quercetin-3-O- $\beta$-D-glucopyranoside & Polygonum cuspidatum & $(31)$ \\
\hline Liquiritinapioside & Glycyrrhizae radix & (30) \\
\hline Silybin & Silybum marianum & (32) \\
\hline Avicularin & Hypericum perforatum & (33) \\
\hline Luteolin-7-O-glucoside & Plantago major & (34) \\
\hline Silymarin & Silybum marianum & (35) \\
\hline Scutallarein & Plantago major & (35) \\
\hline Reynoutrin & Polygonum cuspidatum & (31) \\
\hline
\end{tabular}

Table 2. Lead molecule with protease enzyme of serotype 1

\begin{tabular}{lc}
\hline Flavonoids & $\begin{array}{c}\text { Binding affinity (-kcal/mol) } \\
\text { with serotype 1 }\end{array}$ \\
\hline Amentoflavone & 15.8 \\
Quercetin-3-O- $\beta$-D-glucopyranoside & 15.1 \\
Liquiritinapioside & 15.0 \\
Silybin & 14.7 \\
Avicularin & 14.6 \\
Reynoutrin & 14.6 \\
Luteolin-7-O-glucoside & 14.4 \\
Silymarin & 14.3 \\
Scutallarein & 14.1 \\
\hline
\end{tabular}


183, Lys-181, Phe-180, Gly-201, Asp-179); Luteolin-7-Oglucoside (hydrophobic interactions- His-101, Asn-202, Asp-179, Gly-183, Pro-182, Lys-181, Ser-185, Thr-184 hydrophilic interactions- Val-86, Phe-180, Tyr-200); silymarin (hydrophobic interactions- Val-173, Lys-124, Lys-123, Asp-125, Ala-214, Ala-216, Gly-201 hydrophilic interactions- Gln-217, Ile-215, Asn-202) and scutallarein (hydrophobic interactions- Pro-182, Ser-185, Gly-183, Lys-181, Tyr-200, Tyr-211, Val-212, Gly-203, Ser-213, Leu-178 hydrophilic interactions - Asn-202, Thr-184) were ranked as lead molecules (Figure 1).

Ligplot analysis of lead molecules with protease enzyme of serotype $4(2 \mathrm{VBC})$

Similarly among all the docked flavonoids against 2VBC, amentoflavone (hydrophobic interactions- Tyr-278, Arg217, Arg-254, Arg-215, Gly-255, Leu-212 hydrophilic interactions- Asp-258, His-251, Ile-256, Leu-216) was ranked first followed by silymarin (hydrophobic interactions - Leu-212, Glu-255, Arg-215, Arg-254, Arg217, Pro-276, Tyr-278, His-251 hydrophilic interactionsLeu-216, Ile-256, Asp-258); avicularin (hydrophobic interactions - Ser-328, Arg-463, Leu-193, His-194, Gln327, Pro-326 hydrophilic interactions- Gly-462, Ile461, Pro-195, Gly-459, Ala-316, Ala-455); reynoutrin (hydrophobic interactions - Arg-463, His-194, Ser-328, Pro-326, Leu-193 hydrophilic interactions- Gly-462, Pro195, Gly-459, Ala-455, Asp-192, Gln-327, Ala-316, Ile461); quercetin-3-O- $\beta$-D-glucopyranoside (hydrophobic interactions- Arg-254, Glu-255, Arg-217, Tyr-278, Pro-
Table 3. Lead molecule with protease enzyme of serotype 4

\begin{tabular}{lc}
\hline Flavonoids & $\begin{array}{c}\text { Binding affinity (-kcal/mol) } \\
\text { with serotype 1 }\end{array}$ \\
\hline Amentoflavone & 16.8 \\
Silymarin & 16.0 \\
Avicularin & 15.9 \\
Reynoutrin & 15.9 \\
Quercetin-3-O- $\beta$-D-glucopyranoside & 15.7 \\
Scutallarein & 15.6 \\
Luteolin-7-O-glucoside & 14.4 \\
Silymarin & 14.3 \\
Scutallarein & 14.1 \\
\hline
\end{tabular}

276 hydrophilic interactions- Leu-216, Ile-256, Asp-258, His-251) and Scutallarein (hydrophobic interactions Ser-19, His-41, Leu-65, His-60, Tyr-23, Phe-46, Ile-58, Ile25) were ranked as lead molecules (Table 3 ).

It was evident from the screening results that some flavonoids exhibited noteworthy activities against both serotypes. Plant flavonoids were identified as DENV NS2B/NS3 protease inhibitors. Among all the flavonoids docked, amentoflavone was found to be the lead molecule. So, we procured the amentoflavone from Angene International Ltd., London; tested for DENV NS2B/NS3 protease inhibition and cytotoxicity (Figure 2).

ADMET results

Table 4 shows the in silico ADMET study of lead molecules.
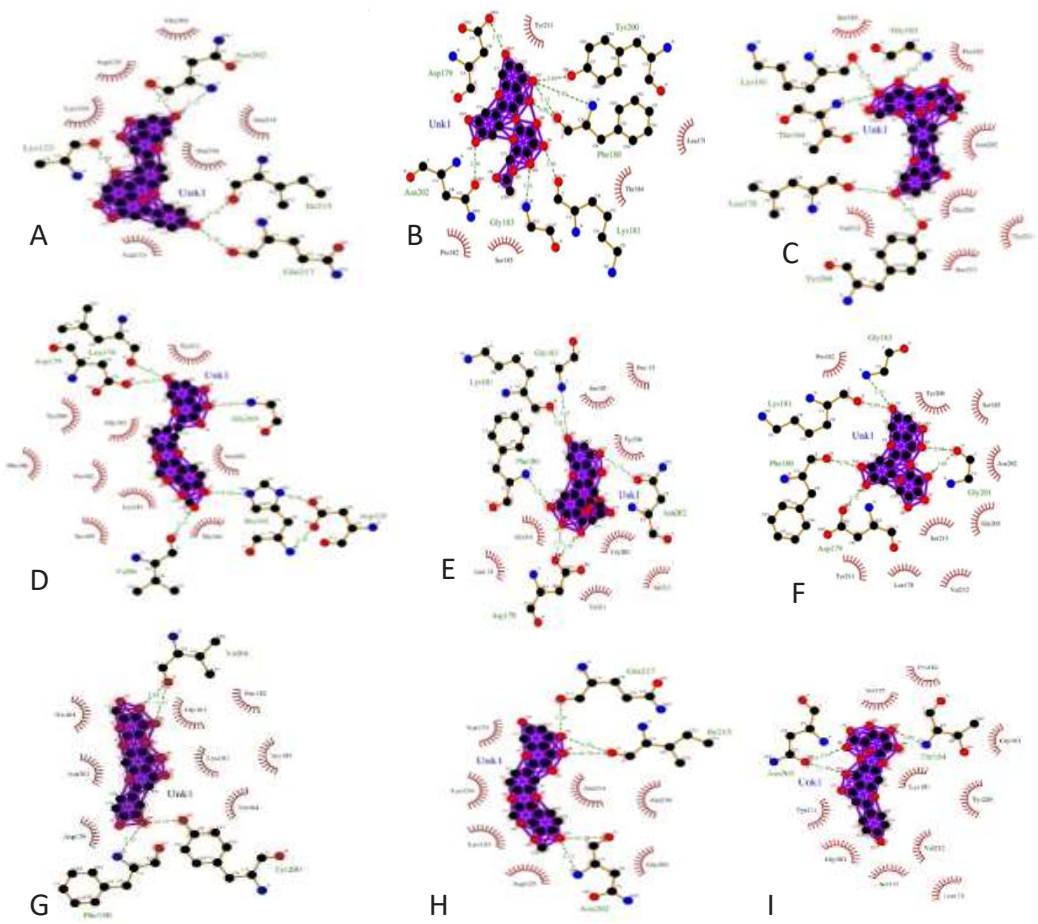

Figure 1. Interactions of lead flavonoids with protease of serotype 1. (A) Amentoflavone, (B) Quercetin-3-O- $\beta$-Dglucopyranoside, (C) Liquiritinapioside (D) Silybin, (E)Avicularin, (F) Reynoutrin, (G) Luteolin-7-O-glucoside, (H) Silymarin and I) Scutallarein. 

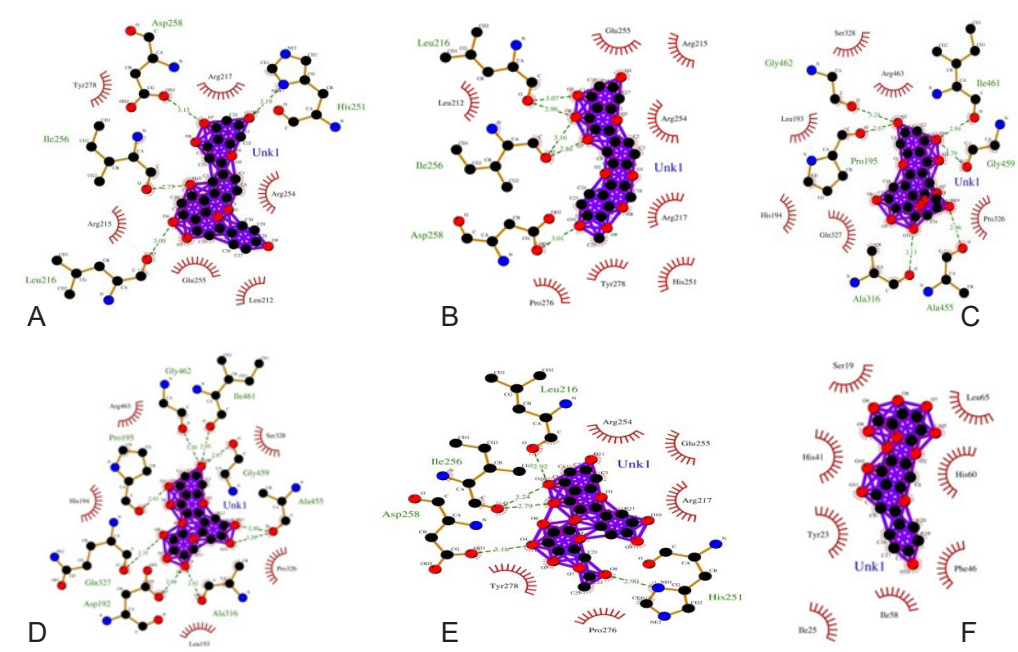

Figure 2. Interactions of lead flavonoids with protease of serotype 4: (A) Amentoflavone, (B) Silymarin, (C) Avicularin, (D) Reynoutrin, (E) Quercetin3-O- $\beta$-D-glucopyranoside, and (F) Scutallarein.

Results indicate that the all lead molecules obtained in the study did not report toxic effect. The lead molecule amentoflavone showed a drug likeliness of 0.21894 and exhibited none toxicity. Silybin reported maximum drug likeliness of 0.58364 and was found to be nontoxic. Lead compounds such as quercetin-3-O- $\beta$-D-glucopyranoside, liquiritinapioside, avicularin, reynoutrin and luteolin7-O-glucoside reported negative score in drug likeliness study.

Protease assay results

The lead molecule amentoflavone was screened for its inhibitory effect on dengue viral protease enzyme. For the protease assay, synthetic peptide bearing dengue protease cleavage site, fluorophore and quencher were used as described above. Purified recombinant protease efficiently cleaved the substrate at NS3 cleavage site and cleaved product exhibited fluorescence. Effect of amentoflavone and standard drug quercetin were measured by the ability of these drugs to decrease the fluorescence. Both amentoflavone and quercetin showed inhibitory activity against dengue viral protease in a dose dependent manner (Figure 3).

Toxicity of Amentoflavone by MTT assay

The cytotoxic effects of amentoflavone and quercetin were screened for their cytotoxic effect using HepG2 cells by MTT cell viability assay. Both compounds showed moderate toxicity against HepG2 cells, however amentoflavone reported less toxicity compared to quercetin (Figure 4).

\section{Discussion}

DENV has four serotypes (37) but any inhibitor against the binding pocket of NS2/NS3 protease is able to work against all the serotypes (38). DENV NS3 protease, as same as other flaviviruses, has been declared as significant drug target. Any disruption in the virus active residues is important, because it may block the replication of virus (39). In the present research, can enable researchers to

Table 4. Drug likeliness and toxicity of the lead flavonoid

\begin{tabular}{|c|c|c|c|c|c|}
\hline Molecule Name & Drug likeness & Mutagenic & Tumorigenic & Reproductive Effective & Irritant \\
\hline Amentoflavone & 0.21894 & None & None & None & None \\
\hline Quercetin-3-O- $\beta$-D-glucopyranoside & -2.1385 & None & None & None & None \\
\hline Liquiritinapioside & -0.1063 & None & None & None & None \\
\hline Silybin & 0.58364 & None & None & None & None \\
\hline Avicularin & -1.6151 & None & None & None & None \\
\hline Reynoutrin & -3.8829 & None & None & None & None \\
\hline Luteolin-7-O-glucoside & -3.2535 & None & None & None & None \\
\hline Silymarin & 0.22481 & None & None & None & None \\
\hline Scutallarein & 0.34679 & None & None & None & None \\
\hline
\end{tabular}




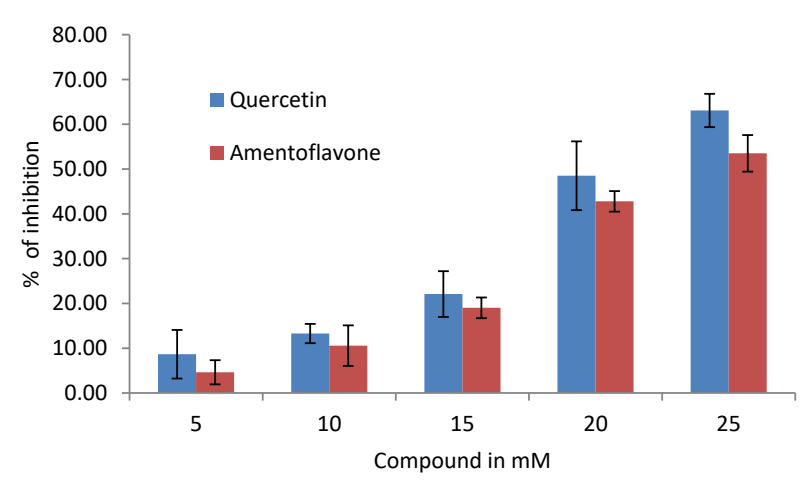

Figure 3. Inhibitory effect of plant extracts against DENV NS3 protease.

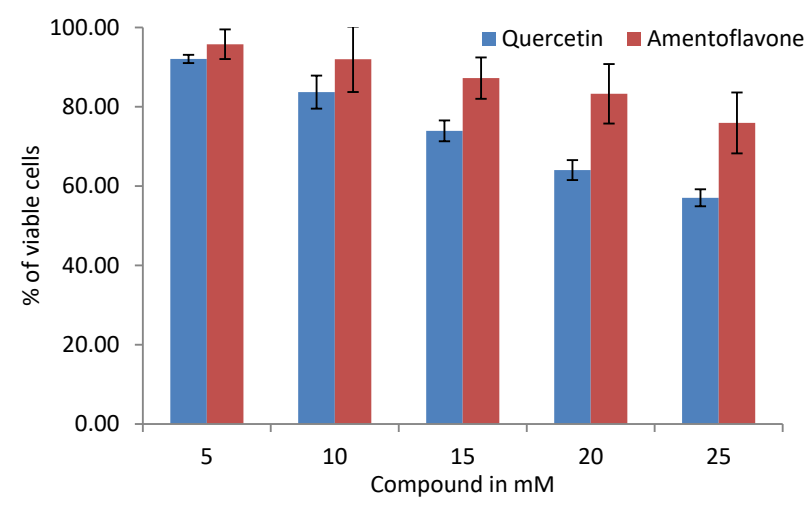

Figure 4. Cytotoxic effect of lead molecule against HepG2 cells.

evaluate the possible binding affinity of various molecules before evaluation in lab. The molecular docking technic is usually used to estimate the binding orientation of various molecules against their targets. In other words, molecular docking is an important technique in drug designing and screening of novel compounds against diseases (27). The current study is focused on the docking of the plant flavonoids against NS2B-NS3 protease of serotype 1 and serotype 4 . Flavonoids were examined for their potency against DENV NS2B-NS3 protease 3L6P and 2VBC of serotype 1 and serotype 2 . In our study, all the listed flavonoids (Table 1) were docked with the active residues of DENV NS2B-NS3 of both serotype 1 and serotype 4 to find their affinity (Table 2 and Table 3 ) as inhibitors. Our results revealed potential and significant interactions of flavonoids with the active residues of the protease.

Among all the flavonoids docked against protease of serotype 1 (3L6P), amentoflavone (-15.8), quercetin-3-O$\beta$-D-glucopyranoside (-15.1), liquiritinapioside (-15.0), silybin (-14.7), avicularin (-14.6), reynoutrin (-14.6), luteolin-7-O-glucoside (-14.4), silymarin (-14.3) and scutallarein (-14.1) were ranked as lead molecules based on their binding energy in $\mathrm{kcal} / \mathrm{mol}$. Similarly among the flavonoids docked against 2VBC, amentoflavone $(-16.8)$, silymarin $(-16.0)$, avicularin $(-15.9)$, reynoutrin
(-15.9), quercetin-3-O- $\beta$-D-glucopyranoside $(-15.7)$ and scutallarein (-15.6) were ranked as lead molecules based on their binding energy in $\mathrm{kcal} / \mathrm{mol}$.

Among all the flavonoids, amentoflavone, quercetin-3-O$\beta$-D-glucopyranoside, avicularin, reynoutrin, silymarin and scutallarein showed potential interaction and significant hydrophobic contact with active residues of both serotypes 1 and 4 .

Amentoflavone was ranked first among all the flavonoids docked against both serotypes (Figure 2A) with protease of serotype 4. Quercetin-3-O- $\beta$-D-glucopyranoside was found at second place interacting with serotype1 (Figure1B) and also found to be potential in inhibiting serotype 4 and placed at fifth position (Figure2E). Liquiritinapioside and silybin were found to interact only with serotype 1 and ranked at third and fourth position (Figure 1C and Figure 1D), respectively but they were not found interacting with serotype 4. Avicularin was found at fifth place interacting with serotype1 (Figure 1E) and also found to be potential by interacting with serotype 4 at third position (Figure 2C). Reynoutrin was also found at fifth place interacting with serotype1 (Figure1F) and was also found to be potential by interacting with serotype 4 at third position (Figure 2E). Silymarin was also found at second place interacting with serotype 4 (Figure 2D) and was also found to be potential by interacting with serotype1 at seventh position (Figure $1 \mathrm{H}$ ). Scutallarein was also found at fifth place interacting with serotype 4 (Figure 2F) and was also found to be potential by interacting with serotype1 at eighth position (Figure 1I). Evaluation of ADMET using in vitro and in vivo assay system is economically not feasible, therefore in silico ADMET methods have been extensively used in various drug discovery programs (40). In our study we used OSIRIS software to predict the ADMET properties of flavonoid. The lead molecule, Amentoflavone had positive drug likeliness and did not show any toxicity. ADMET results of all the molecules used in this study are shown in Supplementary file 1 (Table S2).

A variety of NS3 protease in vitro assays have been developed, utilizing various forms of substrates (41). More recently novel fluorescent based high throughput protease screening assay was developed for screening of hepatitis C virus NS3 serine protease inhibitors. This unique fluorometric assay is very sensitive and has a high throughput capacity making it suitable for screening of potential NS3 serine protease inhibitors. Using such high throughput assay has identified BP2109 as small-molecule inhibitor for the DENV NS2B/NS3 protease (42). In our study we have also used high throughput assay to test protease inhibitory effect of Amentoflavone. Result suggests that amentoflavone has better inhibitory effect compared to standard protease inhibitor quercetin. Toxicity testing is one of the important aspects in antiviral drug discovery. It is expected that, the compounds 
investigating for antiviral effect should be non-toxic to host cells (43). Hence, we have tested the cytotoxic effect of amentoflavone using MTT cell viability assay. Results indicated that amentoflavone had 20\% toxicity ( $80 \%$ viability) at $25 \mu \mathrm{M}$ concentration. Further, the standard compound quercetin showed $40 \%$ toxicity $(60 \%$ viability) at $25 \mu \mathrm{M}$ concentration. It is interesting that the amentoflavone (53\% protease inhibition and $80 \%$ viability at $25 \mu \mathrm{M}$ concentration) appeared to be much more potent in inhibiting the dengue viral protease than quercetin (63\% protease inhibition and $60 \%$ viability at $25 \mu \mathrm{M}$ concentration).

\section{Conclusion}

In this study, amentoflavone was obtained as the lead molecule and a potent inhibitor of dengue viral protease of serotype 1 and serotype 2 . Further, chemical modification and synthesis of analogues could be carried out and used as potential inhibitors against Dengue viral protease.

\section{Acknowledgements}

We thank Dr. Musturi Venkataramana, Department of Biotechnology, University of Hyderabad, Hyderabad for providing the facility to perform protease assay and helpful discussion.

\section{Authors' contributions}

MKJ participated in selection of plants and compounds, as well as in vitro protease assay. PRK participated in docking studies and MTT assay. RYS was involved in research guide planning and execution of research work. ST did ADMET study and PK docking study.

\section{Conflict of interests}

The authors declared there is no conflict of interest.

\section{Ethical considerations}

In the present study we have neither used animal nor human volunteers, hence did not require approval by the ethical committee.

\section{Funding/Support}

No financial support was received for the present work (student and faculty paid the money required for the experiments).

\section{Supplementary Materials}

Supplementary file 1 contains Tables S1-S2.

\section{References}

1. Ganeshkumar P, Murhekar MV, Poornima V, Saravanakumar V, Sukumaran K, Anandaselvasankar A, et al. Dengue infection in India: a systematic review and meta-analysis. PLoS Negl Trop Dis. 2018;12(7):e0006618. doi: $\quad$ 10.1371/journal.pntd.0006618.

2. Guzman A, Istúriz RE. Update on the global spread of dengue. Int J Antimicrob Agents. 2010;36 Suppl 1:S40-2. doi: $\quad 10.1016 /$ j.ijantimicag.2010.06.018.

3. Whitehorn J, Farrar J. Dengue. Br Med Bull. 2010;95:16173. doi: $10.1093 / \mathrm{bmb} / \mathrm{ldq} 019$.

4. Idrees S, Ashfaq UA. A brief review on dengue molecular virology, diagnosis, treatment and prevalence in Pakistan. Genet Vaccines Ther. 2012;10(1):6. doi: 10.1186/14790556-10-6.

5. Hakim ST, Tayyab SM, Qasmi SU, Nadeem SG. An experience with dengue in Pakistan: an expanding problem. Ibnosina J Med Biomed Sci. 2011;3(1):3-8. doi: 10.4103/1947-489x.210848.

6. Weaver SC, Vasilakis N. Molecular evolution of dengue viruses: contributions of phylogenetics to understanding the history and epidemiology of the preeminent arboviral disease. Infect Genet Evol. 2009;9(4):523-40. doi: 10.1016/j. meegid.2009.02.003.

7. Halstead SB. Pathogenesis of dengue: challenges to molecular biology. Science. 1988;239(4839):476-81. doi: $10.1126 /$ science. 3277268 .

8. Chambers TJ, Hahn CS, Galler R, Rice CM. Flavivirus genome organization, expression, and replication. Annu Rev Microbiol. 1990;44:649-88. doi: 10.1146/annurev. mi.44.100190.003245.

9. Rothan HA, Han HC, Ramasamy TS, Othman S, Rahman NA, Yusof R. Inhibition of dengue NS2B-NS3 protease and viral replication in Vero cells by recombinant retrocyclin-1. BMC Infect Dis. 2012;12:314. doi: 10.1186/1471-2334-12314.

10. Idrees $S$, Ashfaq UA. RNAi: antiviral therapy against dengue virus. Asian Pac J Trop Biomed. 2013;3(3):232-6. doi: 10.1016/s2221-1691(13)60057-x.

11. Clarke T. Dengue virus: break-bone fever. Nature. 2002;416(6882):672-4. doi: 10.1038/416672a.

12. Whitehorn J, Simmons CP. The pathogenesis of dengue. Vaccine. 2011;29(42):7221-8. doi: 10.1016/j. vaccine.2011.07.022.

13. Balandrin MF, Klocke JA, Wurtele ES, Bollinger WH. Natural plant chemicals: sources of industrial and medicinal materials. Science. 1985;228(4704):1154-60. doi: 10.1126/ science. 3890182 .

14. Dias DA, Urban S, Roessner U. A historical overview of natural products in drug discovery. Metabolites. 2012;2(2):303-36. doi: 10.3390/metabo2020303.

15. Rates SM. Plants as source of drugs. Toxicon. 2001;39(5):60313.doi:10.1016/s0041-0101(00)00154-9.

16. Wink M. Medicinal Natural Products. A Biosynthetic Approach: P.M. Dewick; Wiley, Chichester, UK, 2002, 2nd ed., 507 pp., £34.95, ISBN 0-471-49641-3. Phytochemistry. 2002;60(6):648. doi: 10.1016/S0031-9422(02)00176-0.

17. Schmidt BM, Ribnicky DM, Lipsky PE, Raskin I. Revisiting the ancient concept of botanical therapeutics. Nat Chem Biol. 2007;3(7):360-6. doi: 10.1038/nchembio0707-360.

18. Saklani A, Kutty SK. Plant-derived compounds in clinical trials. Drug Discov Today. 2008;13(3-4):161-71. doi: 10.1016/j.drudis.2007.10.010.

19. Adams M, Alther W, Kessler M, Kluge M, Hamburger M. Malaria in the renaissance: remedies from European herbals from the 16th and 17th century. J Ethnopharmacol. 2011;133(2):278-88. doi: 10.1016/j.jep.2010.10.060. 
20. Canard B. Full length report on antiviral research and development against dengue virus. 2011. https://www.who. int/tdr/research/ntd/dengue/dengue_full_length_report. pdf.

21. Cordell GA. Sustainable medicines and global health care. Planta Med. 2011;77(11):1129-38. doi: 10.1055/s-00301270731.

22. Tapas AR, Sakarkar DM, Kakde RB. Flavonoids as nutraceuticals: a review. Trop J Pharm Res. 2008;7(3):108999. doi: 10.4314/tjpr.v7i3.14693.

23. Williams RJ, Spencer JP, Rice-Evans C. Flavonoids: antioxidants or signalling molecules? Free Radic Biol Med. 2004;36(7):838-49. doi: 10.1016/j. freeradbiomed.2004.01.001.

24. Singh RP, Gu M, Agarwal R. Silibinin inhibits colorectal cancer growth by inhibiting tumor cell proliferation and angiogenesis. Cancer Res. 2008;68(6):2043-50. doi: 10.1158/0008-5472.can-07-6247.

25. Sung B, Pandey MK, Aggarwal BB. Fisetin, an inhibitor of cyclin-dependent kinase 6 , down-regulates nuclear factorkappaB-regulated cell proliferation, antiapoptotic and metastatic gene products through the suppression of TAK1 and receptor-interacting protein-regulated IkappaBalpha kinase activation. Mol Pharmacol. 2007;71(6):1703-14. doi: 10.1124/mol.107.034512.

26. Garcia-Mediavilla V, Crespo I, Collado PS, Esteller A, Sanchez-Campos S, Tunon MJ, et al. The anti-inflammatory flavones quercetin and kaempferol cause inhibition of inducible nitric oxide synthase, cyclooxygenase- 2 and reactive C-protein, and down-regulation of the nuclear factor kappaB pathway in Chang Liver cells. Eur J Pharmacol. 2007;557(2-3):221-9. doi: 10.1016/j.ejphar.2006.11.014.

27. Lengauer $T$, Rarey M. Computational methods for biomolecular docking. Curr Opin Struct Biol. 1996;6(3):4026. doi: 10.1016/s0959-440x(96)80061-3

28. Ravikumar YS, Ray U, Nandhitha M, Perween A, Raja Naika $\mathrm{H}$, Khanna $\mathrm{N}$, et al. Inhibition of hepatitis $\mathrm{C}$ virus replication by herbal extract: Phyllanthus amarus as potent natural source. Virus Res. 2011;158(1-2):89-97. doi: 10.1016/j.virusres.2011.03.014.

29. Ferrari M, Fornasiero MC, Isetta AM. MTT colorimetric assay for testing macrophage cytotoxic activity in vitro. J Immunol Methods. 1990;131(2):165-72. doi: 10.1016/00221759(90)90187-z.

30. Kang DG, Yin MH, Oh H, Lee DH, Lee HS. Vasorelaxation by amentoflavone isolated from Selaginella tamariscina. Planta Med. 2004;70(8):718-22. doi: 10.1055/s-2004827201.

31. Hayashi H, Hiraoka N, Ikeshiro Y, Yamamoto H. Organ specific localization of flavonoids in Glycyrrhiza glabra L. Plant Sci. 1996;116(2):233-8. doi: 10.1016/0168-
9452(96)04387-7.

32. Minakhmetov RA, Onuchak LA, Kurkin VA, Avdeeva EV, Volotsueva AV. Analysis of flavonoids in Silybum marianum fruit by HPLC. Chem Nat Compd. 2001;37(4):318-21. doi: 10.1023/A:1013758131883.

33. Wu Y, Zhou SD, Li P. Determination of flavonoids in Hypericum perforatum by HPLC analysis. Yao Xue Xue Bao. 2002;37(4):280-2.

34. Samuelsen AB. The traditional uses, chemical constituents and biological activities of Plantago major L. A review. J Ethnopharmacol. 2000;71(1-2):1-21. doi: 10.1016/s03788741(00)00212-9.

35. Katiyar SK, Meleth S, Sharma SD. Silymarin, a flavonoid from milk thistle (Silybum marianum L.), inhibits UVinduced oxidative stress through targeting infiltrating $\mathrm{CD} 11 \mathrm{~b}+$ cells in mouse skin. Photochem Photobiol. 2008;84(2):266-71. doi: 10.1111/j.1751-1097.2007.00241.x.

36. Reina E, Al-Shibani N, Allam E, Gregson KS, Kowolik M, Windsor LJ. The effects of Plantago major on the activation of the neutrophil respiratory burst. J Tradit Complement Med. 2013;3(4):268-72. doi: 10.4103/2225-4110.119706.

37. Melino S, Paci M. Progress for dengue virus diseases. Towards the NS2B-NS3pro inhibition for a therapeuticbased approach. FEBS J. 2007;274(12):2986-3002. doi: 10.1111/j.1742-4658.2007.05831.x.

38. Khan AM, Miotto O, Nascimento EJ, Srinivasan KN, Heiny AT, Zhang GL, et al. Conservation and variability of dengue virus proteins: implications for vaccine design. PLoS Negl Trop Dis. 2008;2(8):e272. doi: 10.1371/journal. pntd.0000272.

39. Li J, Lim SP, Beer D, Patel V, Wen D, Tumanut C, et al. Functional profiling of recombinant NS3 proteases from all four serotypes of dengue virus using tetrapeptide and octapeptide substrate libraries. J Biol Chem. 2005;280(31):28766-74. doi: 10.1074/jbc.M500588200.

40. van Hell AJ, Crommelin DJ, Hennink WE, Mastrobattista E. Stabilization of peptide vesicles by introducing interpeptide disulfide bonds. Pharm Res. 2009;26(9):2186-93. doi: 10.1007/s11095-009-9933-z.

41. Cheng F, Li W, Liu G, Tang Y. In silico ADMET prediction: recent advances, current challenges and future trends. Curr Top Med Chem. 2013;13(11):1273-89. doi: $10.2174 / 15680266113139990033$.

42. Yang CC, Hsieh YC, Lee SJ, Wu SH, Liao CL, Tsao CH, et al. Novel dengue virus-specific NS2B/NS3 protease inhibitor, BP2109, discovered by a high-throughput screening assay. Antimicrob Agents Chemother. 2011;55(1):229-38. doi: 10.1128/aac.00855-10.

43. Spengler U, Lichterfeld M, Rockstroh JK. Antiretroviral drug toxicity -- a challenge for the hepatologist? J Hepatol. 2002;36(2):283-94. doi: 10.1016/s0168-8278(01)00311-7 